\title{
Imaging and Stroke Outcome - Evidence from Upper Austria
}

\author{
By Klaus Buttinger* \\ Harald Stummer
}

\begin{abstract}
Acute Stroke is known as a neurologic emergency, which should be treated as soon as possible. The differences in treatment are dependent on the subtypes of stroke (intracranial hemorrhage/cerebral infarction). To discriminate between bleeding and infarction it is necessary that the patients undergo CT or MRI before treatment. Many recent studies suggest rapid neuroimaging (CT/MRI) for better outcome. However an association between delay of medical imaging, type of imaging and outcome (mortality rate) has not been systematically investigated. The aim of the present paper therefore is to explore the impact of time and type of imaging on outcome. Data was collected over a period of 9 years $(2007$ - 2015) by using clinical data $(N=48,355)$. Binomial logistic regression was used to estimate odds ratios (OR) and 95\% confidence intervals (CI) for the association between age (5 groups) as well as pre-notification by the emergency rescue or doctor, neuroimaging using Stata (C). In several models controlling for patient age and severity of stroke using the National Institutes of Health Stroke Scale (NIHSS), MRI as a diagnostic tool is associated with lower mortality in brain infarction, while for CT imaging no association is found. Time to picture, however, has no significant influence.
\end{abstract}

Keywords: Stroke, Pre-notification, MRI, CT, Austria

\section{Introduction}

Cardiovascular diseases are worldwide leading causes of death. About one third of these deaths is caused by stroke (Bonita, Beaglehole, \& Asplund, 1994) which is the most frequent neurological emergency (Hasenbein, 2006). Due to its high prevalence (Feigin, Lawes, Bennett, Barker-Collo, \& Parag, 2009; Lemesle et al., 1999; Wieberdink, Ikram, Hofman, Koudstaal, \& Breteler, 2012) stroke is one of the most economically important diseases all over the world (Buttinger \& Stummer, 2012).

The diagnosis "stroke" presents with an acute onset of a central nervous deficit on the basis of a vascular pathology, starts suddenly and lasts longer than 24 hours. Fugacious, often self-limiting neurological disorders with a significantly shorter duration are called TIA (Transient Ischemic Attack). In recent years, the concept of "acute ischemic cerebrovascular syndrome" (AICS) is found in the literature (Kidwell \& Warach, 2003). In this concept the duration of the clinical symptoms is not included in the definition.

The two main subtypes of acute stroke (about 15\% cerebral bleeding; about 85\% brain infarction) (Warlow, Sudlow, Dennis, Wardlaw, \& Sandercock, 2003) need different strategies of therapy.

\footnotetext{
${ }^{*}$ Vice Medical Director, Salzkammergut-Klinikum, Austria \& UMIT - Institute for Health Management and Economics, Austria.

${ }^{\dagger}$ Full Professor, UMIT - Institute for Health Management and Economics, Austria \& University Seeburg Castle, Austria.
} 
Dependent from size (mass effect?), location (compression?), age and comorbidity of the patient either conventional therapeutic strategies (slowly resorption of blood) or surgical procedures (evacuation of intracranial blood) are indicated in case of acute stroke due to intracranial hemorrhage (Bösel, Zweckberger, \& Hacke, 2015; Datar \& Rabinstein, 2014; Kirkman, Citerio, \& Smith, 2014).

Different to other sites in the human body the vascular supply of the central nervous system has only few collaterals. In case of a brain infarction at least one (cervical or intracranial) vessel is occluded due to various reasons. This means that in case of a vascular occlusion the affected brain territory gets insufficient blood and oxygen and therefore loses cerebral function and vitality. Because there is only a short time window for survival of brain cells, it is necessary that the blood flow is restored as soon as possible ("time is brain") in order to minimize deaths and improve functional outcomes (Gomez, 2018). Many trials demonstrated that in case of brain infarction intravenous thrombolytic therapy with recombinant tissue plasminogen activator (rtPA) given up to 4.5 hours after symptom onset is an effective treatment which saves lives and improves functional outcome (Adams et al., 2007; Hacke et al., 2008; Hajjar, Kerr, \& Lees, 2011; Lees et al., 2010; Nolte \& Endres, 2012; Schellinger et al., 2007; Shobha, Buchan, Hill, \& Canadian Alteplase for Stroke Effectiveness, 2011). On the other hand outcome data has shown that in less than $30 \%$ of patients with a clot in a proximal intracerebral artery (middle cerebri artery or basilar artery) or in the internal carotid artery intravenous thrombolysis (rtPA) leads to an early recanalization (Fransen et al., 2014).

Therefore in the last years another treatment option for ischemic stroke was proven. Since November 2014 five positive randomized controlled trials (MR CLEAN, REVASCAT, ESCAPE, SWIFT PRIME, EXTEND IA) impressively showed that the treatment of a severe stroke due to a proximal vessel occlusion is safe and highly effective when the blood clot is removed early by mechanical intraarterial thrombectomy (Campbell et al., 2014; Evans, White, Cowley, \& Werring, 2017; Fransen et al., 2014; Molina et al., 2015; Palaniswami \& Yan, 2015; Saver et al., 2015; Weiner \& Ducruet, 2015).

Early intraarterial mechanical thrombectomy (within 6 hours after onset of stroke symptoms) significantly improves outcome in case of severe ischemic stroke (Evans et al., 2017) and therefore this therapeutic procedure has found its way into certain guidelines (Ringleb \& Veltkamp, 2015; Schlaganfall-Gesellschaft, 2017).

A few subgroups of stroke (e.g. wake-up strokes) are not fully covered from these data. In order to select patient groups which will mostly benefit from a mechanical intraarterial thrombectomy a strong need for a multimodal neuroradiologic imaging was shown (Menon, Campbell, Levi, \& Goyal, 2015; Palaniswami \& Yan, 2015).

The DEFUSE3 trial used (CT or MR) perfusion imaging in order to select patients with a severe stroke (6 to 16 hours after a patient was last seen well) eligible for endovascular treatment (Albers et al., 2018). Similar, the DAWN trial used CT (Perfusion) or MR (Diffusion) imaging (difference between infarct 
volume and clinical deficit) in order to select patients suitable for thrombectomy 6 to 24 hours after stroke (Nogueira et al., 2018). Despite of noticeably later beginning of endovascular treatment both trials demonstrated better outcomes for patients treated with endovascular therapy plus standard therapy than standard medical therapy alone. The findings of the DEFUSE3 trial (Albers et al., 2018; Powers et al., 2018) led to a major revision of the 2018 guidelines for the early management of patients with acute ischemic stroke in the US (Powers et al., 2018).

Clinical neurological examinations are used to assess the severity of an acute stroke. For this purpose some scoring systems (clinicometric scales and functional impairment scales) have been developed. The Barthel Index (BI) and the modified Rankin scale (mRS) are widely used functional impairment and disability scales (Ghandehari, 2013). For daily use in emergency situations at hospital sites the National Institute of Health Stroke Scale (NIHSS) has been proven successful. Moreover this deficit scale is also routinely used in clinical stroke trials (Goldstein \& Samsa, 1997).

Many papers describe clinical outcomes from acute stroke and the measurement of clinical outcome parameters after suffering from stroke. Unlike for other diseases, however, there is no conclusive evidence on the right moment and on which kind of measurement (Davalos, Castillo, \& Martinez-Vila, 1995; Duncan, Jorgensen, \& Wade, 2000; Hacke et al., 2008; Johnston et al., 2000; Kotila, Waltimo, Niemi, Laaksonen, \& Lempinen, 1984; Martínez-Vila E, 2004). In addition, different, non-coherent stroke scales (NIHSS, mRS, BI) are used to describe the results of treatment (Ghandehari, 2013; Hacke et al., 2008; Kotila et al., 1984; Ward, Payne, Caro, Heuschmann, \& Kolominsky-Rabas, 2005).

Stroke symptoms are equal in all types of stroke (cerebral bleeding or intracranial infarction) and can only be safely distinguished by neuroradiologic imaging. Computed Tomography (CT) and Magnetic Resonance Imaging (MRI) are practical worldwide used imaging modalities for selecting patients to get the right therapeutic strategy (Adams et al., 2007; Menon et al., 2015; Menon et al., 2013; Veltkamp, 2012)

Noncontrast CT (NCCT) discriminates reliably between cerebral ischemia and intracranial hemorrhage and therefore provides the information to make decisions about therapy options in most of cases (Menon et al., 2015; von Kummer et al., 1994).

CT Angiography (CTA) can easily depict (carotid or intracranial) vessel occlusion and therefore helps clinicians in selecting patients eligible for endovascular treatment (Adams et al., 2007; Menon et al., 2015)

CT Perfusion (CTP) visualizes the infarct core and the tissue at risk. Additional dynamic angiography views generated from raw CTP data are helpful in grading collaterals with excellent temporal resolution (Menon et al., 2013).

MR Diffusion Imaging (DWI), MR Perfusion Imaging (MRP) and MR Angiography (MRA) of patients with acute stroke symptoms are also helpful to rule out stroke mimics and in categorizing the type of stroke (Menon et al., 2015). MR imaging is more time consuming than CT imaging and motion artifacts are more common (Menon et al., 2015; Menon et al., 2013). Therefore, in most 
national guidelines only standard CT or MRI and not Perfusion or Angiography are included.

There is also limited data about outcome from acute stroke and the role of MRI imaging. Burke et al (Burke, Gelb, Quint, Morgenstern, \& Kerber, 2013) looked for circumstances influencing management of patients with acute stroke and outcome parameters in cases where MRI was used (Burke et al., 2013).

If a patient reaches the emergency department with symptoms of an acute stroke quick and robust neuroradiologic imaging (CT or MRI) is necessary for managing further therapeutic steps (Audebert \& Fiebach, 2015; Menon et al., 2015). Time consuming imaging procedures (CTP, MRA, MRP) should only be used in special situations (e.g. wake up stroke) or in controlled stroke trials (Audebert \& Fiebach, 2015).

\section{Aim of the Present Study}

As discussed in the literature review, there is little evidence on the relation between the type of imaging and outcome. The aim of the underlying paper is to analyze more in detail the triangular relation between different imaging types (CT/MRI), treatments used (thrombolysis) and outcomes (mortality) for stroke patients in order to improve clinical practice guidelines. Our proposition for the optimization of the clinical process is the following:

We suggest that (1) a pre-notification (aviso) from the ambulance will reduce the time to image acquisition, (2) the different types of imaging (CT vs. MRI) will lead to differences in treatment and (3) therefore, different outcomes regarding the type of imaging can be observed.

\section{Methodology}

For testing our assumptions, we use a rich stroke registry dataset from Upper Austria containing 48,335 documented insults from 16 hospitals between October 2006 until December 2015. Due to missing data, only 5 of the 9.3 years can be used for the present analysis. Furthermore, we only include cases admitted to the hospitals with one of the following two diagnoses: (i) cerebral infarction (ICD-10 code I63) or (ii) intracranial hemorrhage (ICD-10 code I60-I62). Our primary outcomes include the time to image acquisition measured on a 5-point ordered scale from ( $1=$ image prior to admission, $2=$ less than 0.5 hours, $3=0.5-1$ hour, $4=1-6$ hours to $5=$ image more than 6 hours after admission to the hospital), the probability of receiving thrombolysis $(1=\mathrm{Yes}, 0=\mathrm{No})$ and in-hospital mortality. Secondary outcomes include 7-day mortality, 30-day mortality and 90-day mortality.

In the statistical analysis, a Mann-Whitney $U$ Test is used to compare the median time to imaging acquisition between cases where an aviso happened and cases without aviso. Binomial logistic regressions with hospital fixed effects are used to estimate odds ratios (OR) and 95\% confidence intervals (CI) for (i) the association between MRI, CT and the probability of receiving thrombolysis and 
(ii) MRI, CT and mortality rates controlling for age (measured in years), sex, the stoke severity measured with the National Institutes of Health Stroke Scale (NIHSS) and time to image acquisition for the two main stroke subtypes using Stata 14 (C).

\section{Results}

From the overall database of 9.5 years, only 5 years could be used as all the relevant variables were coded properly and no systematic misses could be detected what reduces the number of cases to 22,199 . As some of the analyses we are referring to only some of the hospitals in the database (e.g. hospitals having both, $\mathrm{CT}$ and MRI) for some of the analyses the number of cases are reduced further down to a lowest number of 3,337.

Our first proposition was that a pre-notification (aviso) from the ambulance or from the emergency doctor significantly reduces the time from admission to image acquisition. Figure 1 shows the distribution of the variable "time to image" by aviso state. When an aviso happened (right panel Figure 1) almost $50 \%$ of patients receive a CT or MRI within the first 30 minutes, whereas without aviso (left panel Figure 1) this proportion drops to less than $30 \%$. Using a Mann-Whitney $U$ test for testing the difference in the two distributions, a significant difference is found between cases with and without pre-notification $(p<0.001)$. Our first result therefore shows that an aviso significantly reduces the time span between admission and image acquisition.

Figure 1. Pre-Notification at the Hospital and Time to Picture

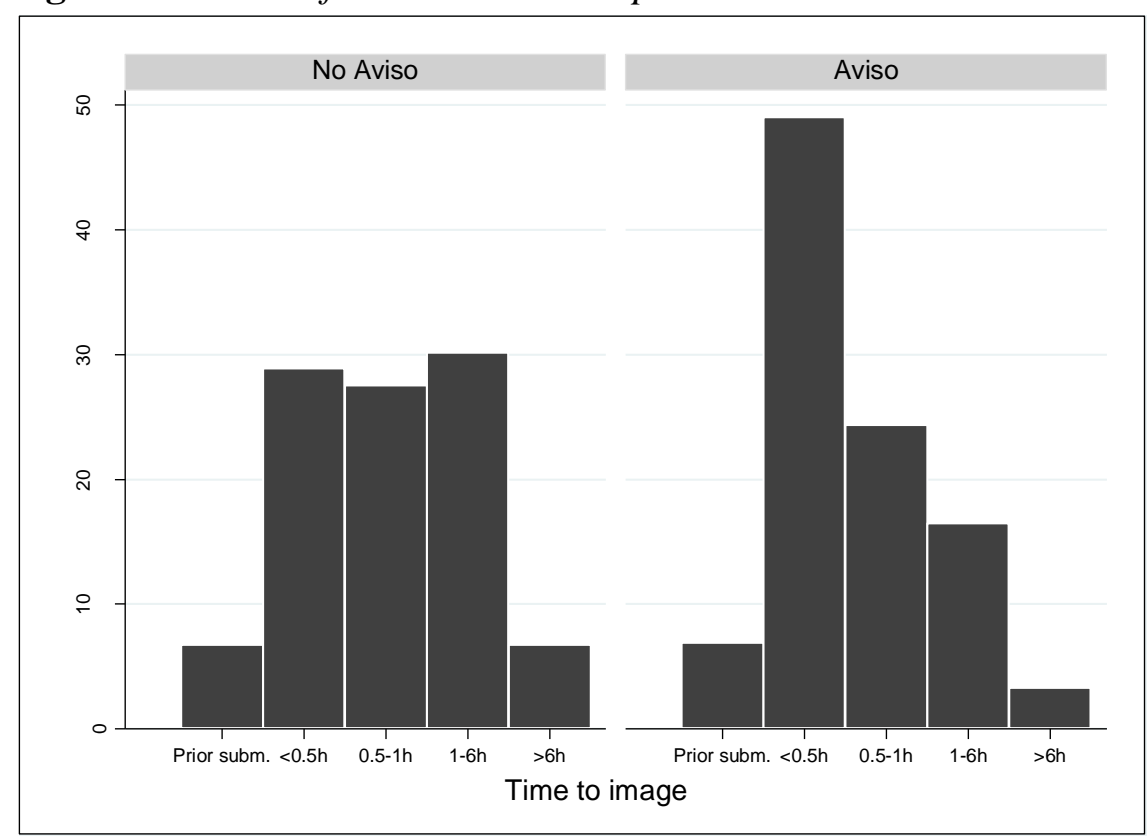

Our second proposition was that using different types of imaging will lead to differences in treatment measured by whether patients receive thrombolysis 
or not. We did this analysis in two steps, testing differently the hospitals that have both, CTs and MRIs and the hospitals only having CTs. In the hospitals having both, 3,583 cases can be analyzed.

Table 1. Influence of Imaging on Thrombolysis Rate within Hospitals Having both, MRI and CT

\begin{tabular}{|l|c|c|}
\hline & \multicolumn{2}{|c|}{ Thrombolysis Rate } \\
\hline Variables & OR (SE) & $\mathbf{9 5 \%}$ CI \\
\hline MRI (=1) & $1.668(0.172)$ & $1.364-2.040$ \\
\hline CT (=1) & $0.382(0.039)$ & $0.313-0.466$ \\
\hline Age (in years) & $0.997(0.004)$ & $0.989-1.004$ \\
\hline Female (=1) & $1.168(0.115)$ & $0.963-1.416$ \\
\hline Time to image & $0.744(0.032)$ & $0.683-0.810$ \\
\hline NIHSS score & $1.085(0.007)$ & $1.071-1.100$ \\
\hline Constant & $0.357(0.111)$ & $0.194-0.655$ \\
\hline Observations & $\mathbf{3 , 5 8 3}$ & \\
\hline
\end{tabular}

Notes: Binomial logistic regression with hospital fixed effects and robust standard errors (SE) clustered on patient level in parentheses.

Table 1 shows the results for running a logistic regression where the probability of thrombolysis is regressed on both MRI and CT controlling for additional covariates for hospitals having both CT and MRI. The results show that when an MRI is used, the odds of thrombolysis is 1.67 times higher than without MRI ( $\mathrm{p}<0.001)$ whereas when a CT is used the odds of performing a thrombolysis significantly decrease to $0.382(\mathrm{p}<0.001)$ compared to no CT. In addition, Table 1 also shows that for patients with a higher NIHSS score the odds for thrombolysis increase significantly while for patients where the time span between admission and image acquisition is high the odds for thrombolysis decrease significantly.

When testing the frequency of thrombolysis between hospitals with both MRI and CT and hospitals only having CT $(\mathrm{N}=310)$, we observe a slightly higher rate of thrombolysis in hospitals with both CT and MRI (OR=1.297, CI: 0.935-1.798). This result, however, is not significant, therefore the treatment behavior within the first group differs depending on the imaging type used, but the treatment behavior does not differ between hospitals having both imaging types and hospitals only having CT.

As we assumed in proposition (3), the different treatment behavior analyzed in proposition (2) should lead to different outcomes. This should only be true for cerebral infarction, as bleeding can be seen in both, CT and MRI. To test our proposition, we run logistic regressions for in-hospital mortality, as well as 7-day, 30-day and 90-day mortality. 
Table 2. Influence of Imaging on In-Hospital Mortality for Hospitals Having both, MRI and CT for Cerebral Infarction (ICD-10 I63)

\begin{tabular}{|l|c|c|}
\hline & \multicolumn{2}{|c|}{ In-hospital mortality } \\
\hline Variables & OR (SE) & $\mathbf{9 5 \%}$ CI \\
\hline CT $(=1)$ & $0.913(0.214)$ & $0.576-1.446$ \\
\hline MRI (=1) & $0.458(0.114)$ & $0.281-0.745$ \\
\hline Age (in years) & $1.068(0.010)$ & $1.049-1.088$ \\
\hline Female (=1) & $0.792(0.139)$ & $0.561-1.118$ \\
\hline Time to image & $0.972(0.085)$ & $0.819-1.154$ \\
\hline NIHSS score & $1.192(0.013)$ & $1.167-1.217$ \\
\hline Constant & $0.0001(0.00008)$ & $0.00002-0.0005$ \\
\hline Observations & $\mathbf{3 , 3 3 7}$ & \\
\hline
\end{tabular}

Notes: Binomial logistic regression with hospital fixed effects and robust standard errors (SE) clustered on patient level in parentheses

Table 2 shows the results for regressing in-hospital mortality on the two imaging types for hospitals having both MRI and CT and for patients diagnosed with cerebral infarction. Controlling for additional covariates, the results show that running an MRI is associated with a significantly lower in-hospital mortality $(\mathrm{OR}=0.458$, CI: 0.281-0.745), whereas for CT we do not find any significant relation. Interestingly, time to image - though a critical variable in guidelines - has no influence on mortality (Table 3 ).

Table 3. Influence of Time to Picture I60-62

\begin{tabular}{|l|c|c|c|c|}
\hline Variables & $\mathbf{( 1 )}$ & $\mathbf{( 2 )}$ & $\mathbf{( 3 )}$ & $\mathbf{( 4 )}$ \\
\hline Age & $\begin{array}{c}\text { In-hospital } \\
\text { mortality }\end{array}$ & $\begin{array}{c}\mathbf{7 - d a y} \\
\text { mortality }\end{array}$ & $\begin{array}{c}\mathbf{3 0 - d a y} \\
\text { mortality }\end{array}$ & $\begin{array}{c}\mathbf{9 0 - d a y} \\
\text { mortality }\end{array}$ \\
\hline in years) & $1.044 * * *$ & 1.031 & $1.037 * *$ & $1.054 * * *$ \\
Female (=1) & $(0.0161)$ & $(0.0196)$ & $(0.0164)$ & $(0.0161)$ \\
\hline Time to & 1.032 & 1.182 & 1.178 & 1.046 \\
picture & $(0.350)$ & $(0.531)$ & $(0.418)$ & $(0.342)$ \\
\hline
\end{tabular}

Notes: Binomial logistic regression with hospital fixed effects and robust standard errors (SE) clustered on patient level in parentheses

When running the same regressions for our secondary outcomes (7-day, 30day, 90-day mortality), the results remain robust (Tables A1-A3 in the Appendix).

In sum, our results show that (1) a pre-notification has a significant influence on time to image, (2) for hospitals having both, CT and MRI, using MRI leads to a significantly higher thrombolysis rate and (3) therefore using MRI (when having a cerebral infarction), is associated to lower mortality rates. This can be due to higher thrombolysis as maybe the doctors feel more secure, but that cannot be proved with the data (4) However, time to image has no impact on mortality rates. 


\section{Discussion}

The two main causes of acute stroke (intracranial bleeding vs. cerebral ischemia) need different therapeutic strategies: Small Hemorrhages without brain herniation disappear by phagozytosis, larger bleedings often need to be evacuated (craniectomy). In case of cerebral ischemia rapid reperfusion techniques (i.v. thrombolysis, endovascular therapy) are necessary in order to supply the brain with blood again.

In acute stroke non-contrast Computed Tomography (NCCT) helps to distinguish intracranial bleeding from cerebral ischemia and is often followed by CT-Angiography in order to select patients with a proximal occlusion of a large intracranial vessel for endovascular treatment within the first 6 hours (Audebert \& Fiebach, 2015).

If there is no information about the beginning of the symptoms (e.g. wake up stroke) MRI (DWI, Perfusion) is used to distinguish between infarct core and penumbra areas (mismatch imaging) (Berkefeld \& Neumann-Haefelin, 2009). On this way patients eligible for endovascular therapy can be selected properly.

Head CT is less time consuming than performing MRI of the neurocranium and enables door-do-needle time of 20 minutes or less to stroke patients (i.v. Thrombolysis). On the other hand MRI is more sensitive in detecting cerebral ischemia and penumbral tissue loss.

What we expected was the positive impact of pre-announcement on the time to the imaging (CT or MRI). This is in line with the literature and the rational expectations. However, there is no significant correlation between time to picture and mortality. Maybe this can be that the time span can really be longer as discussed or mortality is the wrong outcome indicator for this improvement of processes. Using the Barthel-index (Schlote, Krüger, Topp, \& Wallesch, 2004), a nursing based index on activities of daily living would probably either support this result or lead to different results. The latter proposition would be suggested by us.

We tested treatment behavior in hospitals having both, MRI and CT and in hospitals only with a CT. While between those two groups, no significant differences exist, there are significant differences within the group of the hospitals having both. In the latter hospitals, treatment behavior of MDs differs as a function of imaging. Probably, if they are used to having images more sensitive in detecting cerebral ischemia, physicians feel more secure having MRI and are more cautious in giving lysis i.v. if they are not really sure. In opposite to a recent study (Hansen et al., 2018) our findings demonstrate lower mortality rates when MR-Imaging was performed. These different results could be subject for further research.

In the long run, due to additional reperfusion techniques (mechanical thrombectomy) (Evans et al., 2017), guidelines will change, however in many countries this will take some time and then (CT- or MR-) Angiography will be the standard and then those differences should no longer be the case. 


\section{Conclusion}

In hospitals equipped with two imaging modalities (CT and MRI), using MRI leads to a significantly higher thrombolysis rate and is associated with lower mortality rates. Though pre-notification significantly lowers time to image, it does not influence mortality in patients with acute stroke. Retrospective real-world data analysis can - due to high specificity - complement insights from clinical data (Geisel-Marbaise \& Stummer, 2010), however it cannot replace it. Our data show a significant difference in treatment behavior based on imaging modalities more sensitive for detecting cerebral ischemia. Prospective studies however should prove the causality.

\section{Limitations}

Due to data quality issues only part of the database could be used. In future research, better standardized routine data from stroke registers should be able to compare pairs of patients with additional data on activities of daily living.

\section{Ethical Approval}

An approval by a local ethic committee was not necessary due to the local hospital act regarding the use of non-interventional standard data; however the use of the data was registered by the federal data protection commission, the predecessor of the data protection authority domiciled at the federal chancellery under the number DVR 0023981.

\section{References}

Adams HP, Jr., del Zoppo G, Alberts MJ, Bhatt DL, Brass L, Furlan A, et al. (2007) Guidelines for the early Management of Adults with ischemic Stroke: A Guideline from the American Heart Association/American Stroke Association Stroke Council, Clinical Cardiology Council, Cardiovascular Radiology and Intervention Council, and the Atherosclerotic Peripheral Vascular Disease and Quality of Care Outcomes in Research Interdisciplinary Working Groups: The American Academy of Neurology Affirms the Value of this Guideline as an Educational Tool for Neurologists. Circulation 115(20), e478-534.

Albers GW, Marks MP, Kemp S, Christensen S, Tsai JP, Ortega-Gutierrez S, et al. (2018) Thrombectomy for Stroke at 6 to 16 Hours with Selection by Perfusion Imaging. New England Journal of Medicine 378(8): 708-718.

Audebert HJ, Fiebach JB (2015) Brain Imaging in acute Ischemic Stroke-MRI or CT? Current Neurology Neuroscience Reports 15(3): 6.

Berkefeld J, Neumann-Haefelin T (2009) Diagnosis of cerebral Ischemia: When CT and when MRI?. Radiologe 49(4): 299-304.

Bonita R, Beaglehole R, Asplund K (1994) The Worldwide Problem of Stroke. Current Opinion in Neurology 7(1): 5-10. 
Burke JF, Gelb DJ, Quint DJ, Morgenstern LB, Kerber KA (2013) The Impact of MRI on Stroke Management and Outcomes: A Systematic Review. Journal of Evaluation in Clinical Practice 19(6): 987-993.

Buttinger K, Stummer H (2012) Integrierte Schlaganfallversorgung in Oberösterreich [Integrated stroke care in Upper Austria] HeilberufeScience 3(2): 86-92.

Bösel J, Zweckberger K, Hacke W (2015) Haemorrhage and Hemicraniectomy: Refining Surgery for Stroke. Current Opinion in Neurology 28(1): 16-22.

Campbell BC, Mitchell PJ, Yan B, Parsons MW, Christensen S, Churilov L, et al. (2014) A Multicenter, Randomized, Controlled Study to Investigate EXtending the Time for Thrombolysis in Emergency Neurological Deficits with Intra-Arterial therapy (EXTEND-IA). Internation Journal of Stroke 9(1): 126-132.

Datar S, Rabinstein AA (2014) Cerebellar Hemorrhage. Neurology Clinics 32(4): $993-$ 1007.

Davalos A, Castillo J, Martinez-Vila E (1995) Delay in Neurological Attention and Stroke Outcome. Stroke 26(12): 2233-2237.

Duncan PW, Jorgensen HS, Wade DT (2000) Outcome Measures in Acute Stroke Trials: A Systematic Review and Some Recommendations to Improve Practice. Stroke 31(6): 1429-1438.

Evans MRB, White P, Cowley P, Werring DJ (2017) Revolution in Acute Ischaemic Stroke care: A Practical Guide to mechanical Thrombectomy. Practical Neurology 17(4): 252-265.

Feigin VL, Lawes CM, Bennett DA, Barker-Collo SL, Parag V (2009) Worldwide Stroke Incidence and early Case Fatality Reported in 56 Population-based Studies: A Systematic Review. Lancet Neurology 8(4): 355-369.

Fransen PS, Beumer D, Berkhemer OA, van den Berg LA, Lingsma H, van der Lugt A, et al. (2014) MR CLEAN, A Multicenter Randomized Clinical Trial of Endovascular Treatment for acute Ischemic Stroke in the Netherlands: study Protocol for a Randomized Controlled Trial. Trials 15: 343.

Geisel-Marbaise S, Stummer H (2010). Diabetes Adherence-does Gender Matter? Journal of Public Health 18(3): 219-226.

Ghandehari K (2013) Challenging Comparison of stroke Scales. Journal of Research in Medical Science 18(10): 906-910.

Goldstein LB, Samsa GP (1997) Reliability of the National Institutes of Health Stroke Scale. Extension to Non-neurologists in the Context of a Clinical Trial. Stroke 28(2): 307-310.

Gomez CR (2018) Time Is Brain: The Stroke Theory of Relativity. Journal of Stroke and Cerebrovascular Disease 27(8): 2214-2227.

Hacke W, Kaste M, Bluhmki E, Brozman M, Davalos A, Guidetti D, et al. (2008) Thrombolysis with Alteplase 3 to 4.5 Hours after Acute ischemic Stroke. The New England Journal of Medicine 359(13): 1317-1329.

Hajjar K, Kerr DM, Lees KR (2011) Thrombolysis for acute Ischemic Stroke. Journal Vascular Surgery 54(3): 901-907.

Hansen CK, Christensen A, Rodgers H, Havsteen I, Kruuse C, Christensen H (2018) Does the Primary Imaging Modality-Computed Tomography or Magnetic Resonance Imaging-Influence Stroke Physicians' Certainty on whether or not to Give Thrombolysis to Randomized Acute Stroke Patients? Journal of Stroke Cerebrovascular Disease 27(4): 926-935.

Hasenbein U (2006). Leitlinienkonformes Praxiswissen am Beispiel Schlaganfall [Guideline-compliant practical Knowledge using the Example of Stroke]. Deutsches Ärzteblatt 103(24): 1672-1679. 
Johnston KC, Connors AF, Jr, Wagner DP, Knaus WA, Wang X-Q, Haley EC, Jr. (2000) A Predictive Risk Model for Outcomes of Ischemic Stroke. Stroke 31(2): 448-455.

Kidwell CS, Warach S (2003) Acute Ischemic Cerebrovascular Syndrome: Diagnostic Criteria. Stroke 34(12): 2995-2998.

Kirkman MA, Citerio G, Smith M (2014) The intensive Care Management of Acute ischemic Stroke: An Overview. Intensive Care Medicine 40(5): 640-653.

Kotila M, Waltimo O, Niemi ML, Laaksonen R, Lempinen M (1984) The Profile of Recovery from Stroke and factors Influencing Outcome. Stroke 15(6): 1039-1044.

Lees KR, Bluhmki E, von Kummer R, Brott TG, Toni D, Grotta JC, et al. (2010) Time to Treatment with intravenous Alteplase And Outcome in Stroke: An Updated Pooled Analysis of ECASS, ATLANTIS, NINDS, and EPITHET trials. Lancet 375(9727): 1695-1703.

Lemesle M, Milan C, Faivre J, Moreau T, Giroud M, Dumas R (1999) Incidence Trends of Ischemic Stroke and transient Ischemic Attacks in a Well-defined French Population from 1985 through 1994. Stroke 30(2): 371-377.

Martínez-Vila EIP (2004) The Cost of Stroke. Cerebrovascular Disease 17(1): 124-129.

Menon BK, Campbell BC, Levi C, Goyal M (2015) Role of Imaging in current Acute Ischemic Stroke Workflow for Endovascular Therapy. Stroke 46(6): 1453-1461.

Menon BK, O'Brien B, Bivard A, Spratt NJ, Demchuk AM, Miteff F, et al. (2013) Assessment of Leptomeningeal Collaterals using Dynamic CT Angiography in Patients with Acute Ischemic Stroke. Journal of Cerebral Blood Flow \& Metabolism 33(3): 365-371.

Molina CA, Chamorro A, Rovira À, de Miquel A, Serena J, Roman LS, et al. (2015) REVASCAT: A Randomized Trial of Revascularization with SOLITAIRE FR Device vs. best Medical Therapy in the Treatment of Acute Stroke due to Anterior Circulation Large Vessel Occlusion Presenting within Eight-hours of symptom Onset. International Journal of Stroke 10(4): 619-626. doi:10.1111/ijs.12157

Nogueira RG, Jadhav AP, Haussen DC, Bonafe A, Budzik RF, Bhuva P, et al. (2018) Thrombectomy 6 to 24 Hours after Stroke with a Mismatch between Deficit and Infarct. New England Journal of Medicine 378(1): 11-21.

Nolte CH, Endres M (2012) Management of acute Ischemic Stroke. Internist (Berl) 53(5): 585-592.

Palaniswami M, Yan B (2015) Mechanical Thrombectomy Is Now the Gold Standard for Acute Ischemic Stroke: Implications for Routine Clinical Practice. Interval Neurology 4(1-2): 18-29.

Powers WJ, Rabinstein AA, Ackerson T, Adeoye OM, Bambakidis NC, Becker K, et al. (2018) 2018 Guidelines for the Early Management of Patients with Acute Ischemic Stroke: A Guideline for Healthcare Professionals from the American Heart Association/American Stroke Association. Stroke 49(3).

Ringleb A, Veltkamp R (2015) Akuttherapie des ischämischen Schlaganfalls - Ergänzung 2015 [Acute therapy of ischemic stroke - Supplement]. In: German Society of Neurology.

Saver JL, Goyal M, Bonafe A, Diener HC, Levy EI, Pereira VM, et al. (2015) SolitaireTM with the Intention for Thrombectomy as Primary Endovascular Treatment for Acute Ischemic Stroke (SWIFT PRIME) Trial: Protocol for a randomized, Controlled, Multicenter study Comparing the Solitaire Revascularization Device with IV tPA with IV tPA Alone in Acute ischemic Stroke. International Journal of Stroke 10(3): 439-448.

Schellinger PD, Thomalla G, Fiehler J, Köhrmann M, Molina CA, Neumann-Haefelin T, et al. (2007) MRI-based and CT-based Thrombolytic Therapy in Acute Stroke within 
and Beyond Established Time Windows: An Analysis of 1210 Patients. Stroke 38(10): 2640-2645.

Schlaganfall-Gesellschaft Ö (2017) Positionspapier der ÖGSF - Update zu Thrombolyse, Thrombektomie und Antikoagulation [Position paper of the ÖGSF - Update on thrombolysis, thrombectomy and anticoagulation]. In Neurologisch, Supplementum 3/2017: 1-12.

Schlote A, Krüger J, Topp H, Wallesch CW (2004) Inter-rater Reliability of the Barthel Index, the Activity Index, and the Nottingham Extended Activities of Daily Living: The use of ADL Instruments in Stroke Rehabilitation by Medical and Non Medical Personnel Die Rehabilitation 43(2): 75-82.

Shobha N, Buchan AM, Hill MD, Canadian Alteplase for Stroke Effectiveness S (2011) Thrombolysis at 3-4.5 Hours after Acute Ischemic Stroke Onset--Evidence from the Canadian Alteplase for Stroke Effectiveness Study (CASES) registry. Cerebrovascular Disease 31(3): 223-228.

von Kummer R, Meyding-Lamadé U, Forsting M, Rosin L, Rieke K, Hacke W, et al. (1994) Sensitivity and Prognostic Value of Early CT in Occlusion of the Middle Cerebral Artery Trunk. AJNR American Journal of Neuroradiology 15(1): 9-15; discussion 16-18.

Ward A, Payne KA, Caro JJ, Heuschmann PU, Kolominsky-Rabas PL (2005) Care needs and Economic Consequences after acute Ischemic Stroke: The Erlangen Stroke Project. European Journal of Neurology 12(4): 264-267.

Warlow C, Sudlow C, Dennis M, Wardlaw J, Sandercock P (2003) Stroke. Lancet 362(9391): 1211-1224.

Weiner GM, Ducruet AF (2015) ESCAPE Trial Supports Rapid Endovascular Thrombectomy in the Management of Large-Vessel Acute Ischemic Stroke. Neurosurgery 76(6): N15-16.

Wieberdink RG, Ikram MA, Hofman A, Koudstaal PJ, Breteler MM (2012) Trends in Stroke Incidence Rates and Stroke Risk Factors in Rotterdam, the Netherlands from 1990 to 2008. European Journal of Epidemiology 27(4): 287-295. 


\section{Appendix}

Table A1. Influence of Imaging on 7-Day Mortality for Hospitals Having both, MRI and CT for Cerebral Infarction (ICD-10 I63)

\begin{tabular}{|l|c|c|}
\hline \multicolumn{1}{|c|}{} & \multicolumn{2}{|c|}{ 7-day mortality } \\
\hline Variables & OR (SE) & $\mathbf{9 5 \%}$ CI \\
\hline CT $(=1)$ & $0.766(0.200)$ & $0.460-1.277$ \\
\hline MRI (=1) & $0.406(0.121)$ & $0.227-0.727$ \\
\hline Age (in years) & $1.061(0.012)$ & $1.037-1.085$ \\
\hline Female (=1) & $0.821(0.180)$ & $0.535-1.261$ \\
\hline Time to image & $1.061(0.102)$ & $0.879-1.281$ \\
\hline NIHSS score & $1.192(0.014)$ & $1.164-1.220$ \\
\hline Constant & $0.00009(0.00009)$ & $0.00001-0.0006$ \\
\hline Observations & $\mathbf{3 , 3 3 7}$ & \\
\hline
\end{tabular}

Notes: Binomial logistic regression with hospital fixed effects and robust standard errors (SE) clustered on patient level in parentheses.

Table A2. Influence of Imaging on 30-Day Mortality for Hospitals Having both, MRI and CT for Cerebral Infarction (ICD-10 I63)

\begin{tabular}{|l|c|c|}
\hline & \multicolumn{2}{|c|}{ 30-day mortality } \\
\hline Variables & OR (SE) & $\mathbf{9 5 \%}$ CI \\
\hline CT $(=1)$ & $0.978(0.211)$ & $0.641-1.492$ \\
\hline MRI $(=1)$ & $0.443(0.099)$ & $0.285-0.687$ \\
\hline Age (in years) & $1.079(0.010)$ & $1.059-1.100$ \\
\hline Female (=1) & $0.810(0.133)$ & $0.587-1.119$ \\
\hline Time to image & $1.070(0.090)$ & $0.907-1.261$ \\
\hline NIHSS score & $1.185(0.012)$ & $1.161-1.209$ \\
\hline Constant & $0.00006(0.00005)$ & $0.00001-0.0003$ \\
\hline Observations & $\mathbf{3 , 3 3 7}$ & \\
\hline
\end{tabular}

Notes: Binomial logistic regression with hospital fixed effects and robust standard errors (SE) clustered on patient level in parentheses.

Table A3. Influence of Imaging on 90-Day Mortality for Hospitals Having both, MRI and CT for Cerebral Infarction (ICD-10 I63)

\begin{tabular}{|l|c|c|}
\hline & \multicolumn{2}{|c|}{ 90-day mortality } \\
\hline Variables & OR (SE) & 95\% CI \\
\hline CT $(=1)$ & $0.944(0.179)$ & $0.651-1.368$ \\
\hline MRI (=1) & $0.419(0.085)$ & $0.282-0.622$ \\
\hline Age (in years) & $1.095(0.010)$ & $1.076-1.115$ \\
\hline Female (=1) & $0.980(0.136)$ & $0.747-1.286$ \\
\hline Time to image & $1.101(0.079)$ & $0.956-1.267$ \\
\hline NIHSS score & $1.171(0.011)$ & $1.149-1.192$ \\
\hline Constant & $0.00004(0.00003)$ & $0.0000-0.0002$ \\
\hline Observations & $\mathbf{3 , 3 3 7}$ & \\
\hline
\end{tabular}

Notes: Binomial logistic regression with hospital fixed effects and robust standard errors (SE) clustered on patient level in parentheses. 
